\title{
¿Oportunidades de empleo o nuevas formas de trabajo precario? Los call centers de la Zona Metropolitana de Guadalajara
}

\section{Job oportunities or new ways of precarious work? The call centers on the Guadalajara Metropolitan Area}

doi: http://dx.doi.org/10.32870/

espiral.v24i69.5420

\section{Resumen}

Este trabajo problematiza las características de los empleos del sector terciario, especialmente los de venta o atención a clientes por teléfono, surgidos en los llamados call centers. Estos empleos muestran los cambios recientes en las relaciones laborales, donde la supuesta formalidad y las prestaciones son cuestionadas, pues se trata de una nueva forma de precariedad laboral. Se entrevistó a trabajadores de call centers de la Zona Metropolitana de Guadalajara (ZMG). Se les aplicaron entrevistas semiestructuradas sobre sus condiciones laborales. Los hallazgos muestran que la mayoría de estos empleos no son de base, con contratos individuales, temporales y que pueden concluirse sin responsabilidad para el patrón; que los sindicatos son inexistentes; la intensidad y el control sobre el trabajo es alta; el ritmo lo imponen las máquinas y se genera estrés laboral.

Palabras clave: precariedad laboral, flexibilidad, mercados de trabajo, call centers, sector servicios.

\author{
Elena de la Paz Hernández Águila* \\ Juan José Morales Márquez*•
}

Keywords: job insecurity, flexibility, labor markets, call centers, service sector.

\footnotetext{
- Profesora-Investigadora del Departamento de Estudios Sociourbanos, Universidad de Guadalajara, México._elenadelapaz@hotmail.com

- Profesor-Investigador del Departamento de Estudios Sociourbanos, Universidad de Guadalajara, México. morales_juan@yahoo.com

Fecha de recepción: I4 de abril de 2016. Fecha de aceptación: I3 de octubre de 2016.
} 


\section{Introducción'}

En las últimas tres décadas, México pasó por relevantes transformaciones en sus sectores económicos. La industria manufacturera, que lideró el crecimiento económico durante el periodo del desarrollo estabilizador, muestra ahora un profundo declive, mientras que se observa un importante desarrollo del sector servicios. Debido a este fenómeno, numerosos autores han comenzado a documentar el papel del sector servicios como el nuevo motor de crecimiento de la economía. Garza (2011, p. 351) afirma que el análisis de los servicios es fundamental para comprender la evolución macroeconómica en las últimas décadas del siglo XX y en las primeras del siglo XXI.

Este proceso de terciarización de la economía es un fenómeno complejo, porque dentro del sector servicios se desarrollan actividades tan diversas que los estudiosos no terminan de ponerse de acuerdo sobre los rubros que deben incluirse ni sobre la apropiada denominación del sector.

El trabajo que se presenta aquí tiene como objetivo estudiar sólo una pequeña parte de la complejidad del sector terciario. Interesa documentar el surgimiento de nuevos servicios que parecen ser desarrollados en principio por empresas interesadas en la venta por vía telefónica, o bien en la atención de sus clientes, por dicha vía o a través de Internet. Si bien existen diversos estudios sobre los call centers $^{2}$ en México, como los de Hualde, Tolentino y Jurado (2014), Micheli Thirión (2007 y 2012), Montarcé (2011),

I. Los autores de este trabajo expresamos nuestro agradecimiento al Lic. Jesús Zazueta Borboa, quien como parte de su servicio social colaboró realizando algunas entrevistas para la presente investigación.

2. Los call centers son el soporte desarrollado por las empresas para satisfacer determinados aspectos de la relación con sus clientes en forma no presencial. Son plataformas telefónicas que pueden ser gestionadas directamente por la empresa interesada en brindar atención a sus clientes o por empresas proveedoras, en las que se ha terciarizado su gestión (Del Bono y Bulloni, 2008, p. 3).

\section{6}


Da Cruz y Fouquet (2010), entre otros, estos autores han puesto énfasis en el estudio de las empresas instaladas en las regiones ya sea del norte del país, como Tijuana y Monterrey, o en la Ciudad de México. Sin embargo, una ciudad como Guadalajara, que es la tercera receptora de este tipo de empresas en el país y que se ha caracterizado por el establecimiento de numerosas industrias maquiladoras y un mercado de trabajo con alta composición de industrias tradicionales, no ha sido estudiada en el impacto que los call centers han tenido sobre la fuerza de trabajo local.

Asimismo, este trabajo da pauta para entender la deslocalización de las empresas internacionales y la búsqueda que hacen de regiones y espacios propicios a sus intereses de expansión, obtención de ganancias y oportunidades de crecimiento global, lo cual genera incertidumbre tanto en los trabajadores de los países desarrollados como en aquellos en vías de crecimiento económico y tecnológico (Taylor y Bain, 2005).

El arribo y expansión de lo que algunos especialistas han denominado "las fábricas de la charla" se presenta como un fenómeno novedoso y creciente en diversos países de América Latina, especialmente Brasil (Rosenfield, 2007), Argentina (Lisdero, 2009, y Viadana, et al., 2010), Chile (Escobar Salazar, 2012) y México (Hualde, et al., 2011; Micheli Thirión, 2007; Montarcé, 2011), lo que amerita que el fenómeno sea estudiado para encontrar las especificidades en cada nación.

En este trabajo, se analiza el caso de México, particularmente la Zona Metropolitana de Guadalajara (ZMG), y las preguntas que se pretenden responder son: ¿cómo se configuran las relaciones laborales en los nuevos empleos del sector servicios en la ZMG?, y ¿se puede considerar que los empleos que se generan por los call centers responden a nuevas formas de trabajo precario? 
Para responder estas preguntas, se utilizó una metodología de tipo cualitativo: se entrevistó a trabajadores de ambos sexos de entre 18 y 30 años de edad, la mayoría de ellos estudiantes de licenciatura de escuelas de la ZMG, mayormente de la Universidad de Guadalajara y del Instituto Tecnológico de Estudios Superiores de Occidente (ITESO). La selección de la muestra de trabajo fue a través del método de la bola de nieve, donde los entrevistados referenciaban a otros trabajadores y donde el corte del trabajo de campo se dio bajo el método de saturación o repetición de las respuestas obtenidas. La selección de los entrevistados se realizó considerando los diversos tipos de call centers que existen en la localidad; los criterios de inclusión consideraron: a) si la empresa presta servicios solamente en español o también en otro idioma, b) el tamaño del call center considerando su número de trabajadores, y c) el origen nacional o extranjero de la empresa.

De acuerdo a las respuestas obtenidas y a los postulados teóricos que defiende el trabajo, las entrevistas sirvieron para mostrar cómo los sujetos viven y recrean en lo cotidiano la precariedad y los cambios que se viven en el actual mundo laboral, donde la vida diaria y sus problemas son reflejo de las modificaciones y cambios de estos mercados laborales, y donde las diferencias entre las respuestas abonan a la complejidad del fenómeno estudiado y muestran lo heterogéneo del ser social ante el embate de las nuevas formas de empleo del capitalismo actual.

El trabajo quedó integrado en cuatro apartados. En el primero se describe la llegada de los call centers al estado de Jalisco, en específico a la ZMG, sus orígenes y desarrollo, así como el tipo de servicios que ofrecen y las características del mercado laboral donde se insertan; en el segundo apartado se presenta la discusión teórica sobre el tema de la precariedad laboral, los principales enfoques y la evolución del concepto; en el tercero se problematiza -contrastando 
los hallazgos en campo- si los empleos en estos centros de trabajo se pueden considerar o no como precarios; finalmente, se presentan algunas conclusiones.

\section{El arribo de los call centers}

En los últimos años, de acuerdo a los resultados de la Encuesta Nacional de Ocupación y Empleo, el sector que más empleos genera en Jalisco es el de los servicios, ya que el $64.1 \%$ de la población económicamente activa se ubica en ese sector, el $26.2 \%$ en la industria y el $9.1 \%$ en la agricultura (ENOE, 2013). Asimismo, el sector de los servicios es el más dinámico en cuanto a la creación de empleos y compra de tecnología.

En el caso específico de los call centers, se puede señalar que se instalan en la ciudad de Guadalajara y su zona metropolitana mayoritariamente desde mediados de los años noventa del siglo pasado, al igual que en el resto del país, aprovechando las ventajas que ofrecía la metrópoli para ello, que los atraía por el tipo de mano de obra presente en la ciudad, con un alto grado de educación, una gran cantidad de jóvenes y un bajo costo de los salarios, además de las políticas de apoyo por parte del Gobierno estatal para la llegada de empresas de tecnologías de la información.

Guadalajara y sus municipios conurbados son la segunda zona metropolitana más grande de México en términos de población: suma 4434878 habitantes, de los cuales la población de entre 15 y 29 años representa el 27\%. Es el principal centro económico del occidente del país y la segunda economía en el sector servicios, sólo después de la Ciudad de México. En los últimos años, la ZMG ha promo- 
vido la instalación de una ciudad (parque) digital y de los campus party más grandes de América Latina. ${ }^{3}$

Además, es sede de numerosas universidades privadas y de la segunda universidad pública más grande del país. La presencia de universidades en una ciudad es requisito importante para la instalación de los call centers, que requieren una mano de obra educada y bilingüe; además, en los últimos años se han ofertado una gran cantidad de carreras referidas a las áreas de cómputo y tecnologías de información. Por otra parte, Jalisco destaca como una de las entidades con más alto índice de migrantes del país, y a su vez de retornados. Los migrantes de retorno se han constituido en una fuerza importante de trabajo para estas empresas por su dominio del idioma inglés.

Asimismo, los call centers aprovechan las ventajas de instalarse en un mercado de trabajo caracterizado por alta flexibilidad, inseguridad en el empleo y creciente precariedad. De acuerdo con López Pedroza (2016), el 45\% de los trabajadores de Jalisco laboran sin un contrato escrito, lo que se traduce en empleos inseguros y sin derecho a prestaciones sociales. En lo que se refiere a las remuneraciones, la mayor parte de los asalariados ganan menos de tres o hasta tres salarios mínimos, lo que equivale a vivir con alrededor de MXN 6573.36 mensuales, lo que resulta insuficiente para cubrir las necesidades de una familia dado que el costo de la canasta básica es de MXN 9035.00 (Del Toro, 2016). En este contexto, es explicable la llegada de importantes empresas de call centers a la localidad, que se presentan, en opinión de los empresarios y de algunos trabajadores, como importantes oportunidades de empleo.

3. Un campus party es una reunión de varios días donde se concentran jóvenes con talentos en emprendimiento y tecnología y reciben conferencias y cursos, además de que forman equipos de trabajo que en muchas ocasiones se convierten después del campus en nuevos proyectos o trabajos en conjunto. 
En la ZMG, se encuentran presentes filiales de los call centers más grandes del país, como Teleperformance, Teletech, Atención Telefónica, Sertec, Megacall, Consorcio Jurídico de Cobranza Especializada, Servifon, Asecon, Unifica, Soluciones Telefónicas de Occidente, Billing en Comcast, Anexa, y recientemente AT\&T. ${ }^{4}$

Según fuentes oficiales de la Secretaria del Trabajo del estado de Jalisco, la ZMG cuenta con 6940 estaciones de trabajo y 9125 ejecutivos telefónicos en call centers, y se pronostican muchas posibilidades de seguir atrayendo empresas del ramo a la zona, esto debido principalmente a la saturación que se vive de estos centros de trabajo en la Ciudad de México, el Estado de México y Monterrey ( $E l$ Informador, 2014). ${ }^{5}$

Gráfica 1. Números totales de las estaciones de trabajo y ejecutivos telefónicos de los call centers de la ZMG

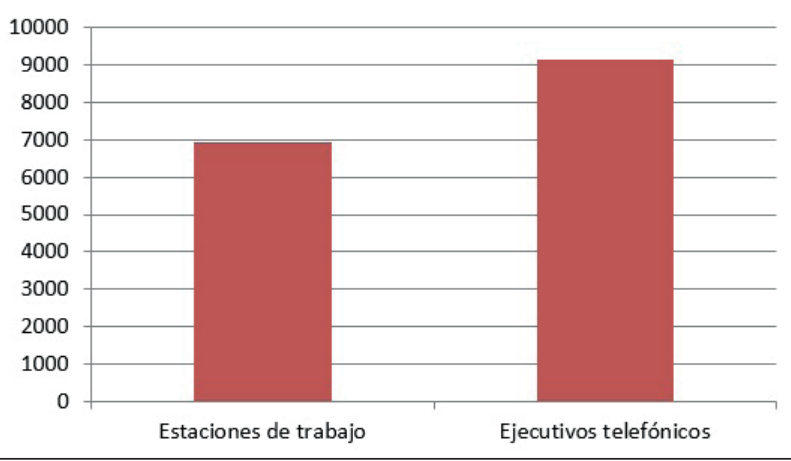

Fuente: elaboración propia con base en El Informador (2014).

4. Este call center es el primero que instala esta empresa en el país y busca ser el de mayor tamaño de la ciudad.

5. La Asociación de Industriales de Occidente considera que el número de los empleos en los call centers en la ZMG asciende a casi 50 000. Los datos de esta investigación se basan en la información oficial de la Secretaria del Trabajo y Previsión Social de Jalisco y del Instituto Mexicano de Teleservicios. No obstante, se considera que puede haber un subregistro debido a la práctica del outsourcing que hacen estas empresas. 
La importancia de Jalisco y la ZMG en la industria de los call centers a nivel nacional es visible con base en los siguientes indicadores: en el 2013, la ZMG tenía el 8.6\% de las estaciones de trabajo, el $9.5 \%$ del total de los empleados y el $6.7 \%$ del total de centros de trabajo (IMT, 2013). La ZMG solamente estaba por debajo de la Ciudad de México, Monterrey y el Estado de México.

Gráfica 2. Importancia nacional de los call centers de la ZMG a nivel nacional (porcentajes)

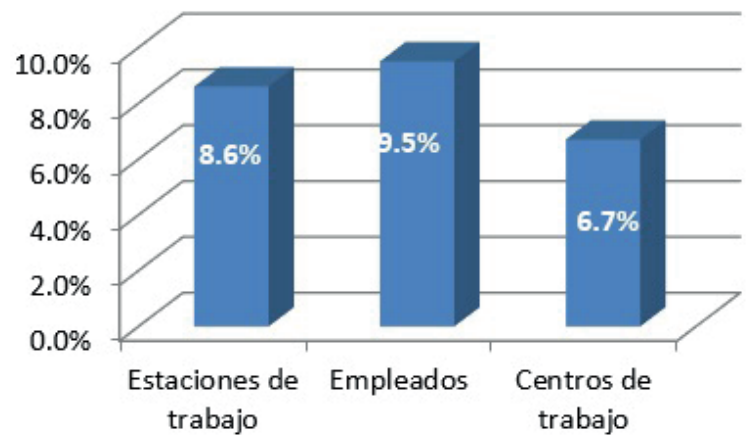

Fuente: elaboración propia con base en IMT (2013).

En su división por empresas, los call centers se pueden separar en in house, que pertenecen a la compañía de la cual promocionan sus servicios, y outsourcing, modelo en el que aparece un tercero que es aquella empresa para la cual los call centers prestan sus servicios. En lo referente a su división interna del trabajo, estas empresas generalmente funcionan como grandes equipos donde se tiene un responsable del mismo, denominado de distintas formas de acuerdo a la empresa como "supervisor", “coach”, "líder”, etc., y que es responsable de un grupo de teleoperadores, es decir, los trabajadores que hacen o reciben las llamadas o los emails. 
Generalmente, varios supervisores dependen de un subgerente y varios de estos de un gerente o director general. Además de las personas que trabajan como supervisores, también se cuenta con departamentos de capacitación y de logística y mantenimiento de los equipos. Algunos call centers sólo tienen sus áreas de trabajo y un pequeño comedor, pero se han encontrado algunos que además del comedor tienen salas de descanso y ocio para sus trabajadores.

En lo que se refiere a los servicios prestados por los call centers de la ZMG, estos no varían mucho con respecto a los que se ofrecen a nivel nacional, es decir, se trabaja principalmente para empresas financieras, compañías telefónicas y de seguros, en actividades de cobranza, ventas, atención a clientes de productos de alta tecnología, banca en línea, etc. (Micheli Thirión, 2012).

Los trabajadores de los call centers comparten una cotidianidad laboral muy semejante, pero también viven condiciones de trabajo heterogéneas. Por ejemplo, aquellos que laboran en empresas que atienden a clientes de otros países en idioma inglés obtienen mejores salarios y prestaciones, circunstancias que, entre otras, hacen que algunos empleados consideren que son una buena oportunidad de trabajo. Sin embargo, en este estudio se pretende mostrar que aun cuando se trata de empleos dentro del sector formal de la economía, el trabajo que se ofrece en los centros de atención se puede considerar precario.

Por lo anterior, toma relevancia recuperar la discusión sobre el concepto de precariedad laboral y su evolución en el caso de América Latina.

\section{La discusión sobre la precariedad laboral en la década de los ochenta}

La reflexión sobre la precariedad laboral se inaugura en Europa a inicios de la década de los ochenta del siglo 
pasado, y de acuerdo a Rodgers y Rodgers (1989) afectó de manera importante a la totalidad de los países europeos, donde generó incertidumbre y desesperación entre los trabajadores, quienes veían cómo llegaba a su fin la época del Estado de bienestar y se acercaba una época de austeridad económica y de precariedad laboral (Rodgers y Rodgers, 1989).

Esta discusión llegó a América Latina hacia la segunda mitad de la misma década. A partir de entonces, mucha tinta ha corrido no sólo para definir lo que se entiende por "trabajo precario", sino para construir indicadores que de cumplirse nos indiquen por qué una actividad puede incluirse dentro de esa categoría. Numerosos autores han abordado el tema; no es un objetivo del trabajo hacer un recuento de ello, pero sí se pretende mencionar cómo ha evolucionado la discusión sobre lo precario desde las distintas miradas de la academia. En ese sentido, el trabajo de Minor Mora puede ser útil dado que realiza un recuento de estas posiciones.

Mora (2005) refiere diversas maneras en que se ha discutido el concepto de trabajo precario: 1) un conjunto de situaciones empíricas relacionadas con la emergencia y expansión de relaciones no formales de trabajo (contratos atípicos, contratos temporales, trabajos estacionales, a destajo, etc.); 2) da cuenta de situaciones en las que las condiciones de trabajo se han deteriorado, ya sea como consecuencia de la caída de los salarios por debajo de los mínimos fijados por la ley, la prolongación de las jornadas laborales, o bien la contratación presente de trabajadores a tiempo parcial; 3) refiere a situaciones en las que el personal es contratado sin acceso a regímenes de seguridad social; y 4) da cuenta de la existencia de relaciones laborales en las que las condiciones de trabajo, la duración de la relación contractual y la provisión de derechos laborales son una prerrogativa empresarial debido tanto a procesos formales 
de cambio en la legislación y nuevas prácticas empresariales como a modelos autoritarios de regulación de las relaciones laborales.

Los enfoques metodológicos para estudiar el fenómeno también son disímbolos: podría decirse que entre algunos académicos predominaron aquellos enfoques que pretendían establecer si un trabajo era o no precario con base en ciertas variables (tipo de contrato, salarios, prestaciones, sólo por mencionar las más comunes en todos los autores).

Otros autores plantearon un proceso de medición más riguroso (Hualde y Serrano, 2005), en donde ya no se trata de contestar con un "sí" o un "no" a la pregunta de la precariedad, sino de establecer los tipos de calidad en el empleo, en una escala de graduación que va de la precariedad a la alta calidad. Hualde y Serrano (2005) sugieren cinco variables para medir la calidad del empleo: 1) pertenencia al mercado de trabajo como asalariado, 2) formas de los contratos de trabajo, 3) prestaciones de ley, 4) horas trabajadas, y 5) ingresos.

No obstante, otros autores consideraron que medir la calidad del empleo también presenta dificultades a la hora de otorgar ponderación a cada indicador que se pretende medir. Por ello, otros enfoques como el de Lindón (2003) proponen explorar la precariedad laboral desde la mirada de quien trabaja. Recrear no sólo el ámbito objetivo, sino también el subjetivo del trabajo, la experiencia de vivir y ser precario.

Un grupo de autores, entre los que se encuentra De Oliveira (2006), se han planteado construir un concepto de precariedad laboral que recupere varias dimensiones: la económica, la normativa y la social, o de seguridad laboral.

Finalmente, cabe señalar que en un trabajo más reciente Guadarrama, Hualde y López (2014) construyen una propuesta más integral que contempla tres ejes de análisis: a) el eje de la heterogeneidad, que considera las diferencias de la 
precariedad laboral por ocupación, región y género y que les permite la comparación a partir de algunos indicadores; b) el eje diacrónico, que es útil para mostrar el carácter históricobiográfico del fenómeno y el carácter no determinista de la precariedad, ya que puede revertirse o profundizarse en determinados contextos familiares, formativos, laborales, regionales y transnacionales, de acuerdo a las trayectorias de vida de las personas; y c) el eje de la subjetividad, que pone el énfasis en la precariedad desde el punto de vista del sujeto que la vive y que alude a los significados que los individuos le otorgan (Guadarrama, Hualde y López, 2014, pp. 27-29).

Si se intenta hacer un recuento de los indicadores más comunes que fueron utilizados en el intento de medir la precariedad en el empleo, se encuentra que estos son tipo de contrato, jornada laboral, ingresos, prestaciones de ley, sindicalización y estabilidad laboral. No obstante, en el conjunto de los trabajos revisados se llegó a utilizar más de una decena de indicadores diferentes a los mencionados con el mismo objetivo.

A pesar de lo controvertido y polisémico que resulta este término y las propuestas metodológicas para su medición, el concepto de precariedad laboral parece conservar cierta vitalidad y continúa en la actualidad siendo utilizado por numerosos investigadores (Arteaga García y Micheli Thirión, 2010). Debe señalarse la necesidad de seguir documentando las profundas transformaciones que el trabajo ha sufrido en las últimas décadas, y de trabajar en la reformulación de algunas categorías para dar cuenta de los nuevos procesos en el mundo del trabajo.

Hacia una nueva definición de la formalidad precaria

El trabajo formal actual es un empleo diferente al tradicionalmente conocido como trabajo clásico, que de acuerdo 
a De la Garza (2011) agruparía a aquellos trabajos que se dieron en la etapa de posguerra, principalmente en países desarrollados y en las grandes empresas de tipo industrial fordista, los cuales entre otras cuestiones ofrecían estabilidad y un buen salario, o que a decir de Zapata (2005) generaban estabilidad y posibilitaban el ascenso y el acceso a toda una serie de prestaciones a las cuales el sector informal no tenía derecho.

Si bien el desarrollo económico de México no se puede comparar al desarrollo de los países centrales de Europa o de América del Norte, en nuestro país durante el modelo de sustitución de exportaciones el apoyo a los empresarios mexicanos y el desarrollo de las instituciones sociales posibilitó que un gran porcentaje de los empleos ubicados en el sector industrial y en la administración pública, creados en esa época, se pudieran considerar de calidad, esto por las prestaciones y las características de los mismos, como salarios dignos, buenas condiciones laborales, existencia de contratos colectivos y sindicalización de sus trabajadores (Zapata, 2005).

La distinción entre formalidad e informalidad en el México actual no provee elementos suficientes para caracterizar la calidad de los empleos, dado que en el nuevo contexto de la reforma a la Ley Federal del Trabajo, aprobada el 13 de noviembre de 2012, al adicionar los artículos 15A y 15D que legalizan el fenómeno de la subcontratación, se permite que los trabajadores pierdan seguridad y prestaciones, aun cuando sus empleos son formales y reconocidos por ley; además, con la reforma al artículo 39 se establecen dos figuras que permiten los contratos por tiempo indeterminado: la capacitación inicial y el periodo de prueba. Así, se observa la proliferación de contratos por ciento veinte y ciento ochenta días que sin dificultad para el patrón pueden ser concluidos. 
De este modo, la precarización del empleo se vuelve un elemento "normal" del nuevo escenario laboral, igual que la flexibilidad en el trabajo, cuya instalación en nuestro país para nada corresponde a la visión "romántica" de la manufactura flexible expuesta por Piore y Sabel (1990) cuando reflexionaron sobre la segunda ruptura industrial.

La flexibilidad moderna en México ha permitido un dejar hacer sin trabas a las gerencias en los procesos de trabajo, suprimiendo seguridades laborales, sindicatos y contrataciones colectivas, todo lo cual es visto como obstáculo a la modernización y a la competitividad.

Así, un nuevo concepto que permitiría explicar estas nuevas formas de trabajo es el de "flexi-precariedad", donde el término hace referencia a la flexibilidad que se requiere en el empleo, cuando en ocasiones se exige una polivalencia del trabajador para ejercer varias funciones, y también refiere a la flexibilidad de la actual fuerza de trabajo para emplearse en las más diversas áreas, pasando de un sector a otro, de una actividad a otra completamente diferente, flexibilidad en la contratación, e incluso en las formas de trabajo y pago de salario, entre otras.

Es decir, en el establecimiento de la flexi-precariedad se implica la participación de los tres grandes actores del mundo del trabajo: el Estado, quien a través de sus leyes proclama las reformas que legalizan de facto las practicas del outsourcing y las contrataciones de tiempo parcial; las empresas, que eluden su responsabilidad como patrones y entre otras políticas promueven empleos donde no se garantiza la continuidad en el mismo; y los sindicatos, que no se hacen presentes o no tienen habilidad de negociación, y en otros casos aceptan la firma de contratos de protección para "sindicalizar". ${ }^{6}$ 
Tomando como base dicha discusión, se pretende realizar un análisis a mayor profundidad sobre las características del mercado de trabajo que ofrecen los call centers, las condiciones generales del empleo y la posible calidad del mismo, esto con la finalidad de contar con elementos que ayuden a comprender si en realidad estos empleos constituyen verdaderas oportunidades de desarrollo y crecimiento para los trabajadores, o sólo son nuevas formas de empleo precario.

\section{Las condiciones de trabajo y las relaciones laborales} de los call centers de la ZMG

\section{La terciarización de la fuerza de trabajo}

Una característica que identifica a varios de los call centers de la ZMG es la inseguridad en el empleo, ya que aunque algunos de ellos ofrecen la definitividad después de varios contratos laborales (generalmente dos), en otros call centers los contratos de trabajo son individuales y por obra determinada, es decir, "trabajan por campañas" y cuando estas concluyen se considera que se agota la materia de trabajo y los teleoperadores pueden ser despedidos.

Los call centers no sólo son empresas subcontratadas, sino que a su vez estas terciarizan la provisión de mano de obra. En algunos de los casos estudiados se pudo detectar que sus trabajadores firmaron su contrato con otra compañía, generalmente agencias de subcontratación.

Entre las empresas cliente y los call centers media una relación jurídica de carácter comercial y no laboral, las primeras tienen un alto nivel de injerencia en la determinación de la actividad y de las relaciones laborales de los operadores de call centers, lo que permite pensar que

7. Se denomina "campaña" al contrato celebrado entre un call center y otra compañía, gracias al cual se promociona un producto por tiempo determinado. 
se trata claramente de una relación de subcontratación laboral y no de una relación meramente comercial entre las mismas (Montarcé, et al., 20I2, pp. 347-348).

Si bien es cierto que los trabajadores de Jalisco desde décadas pasadas - con la llegada de las transnacionales de la industria electrónica- padecen de la práctica cada vez más frecuente de la subcontratación por agencias, en el caso de los call centers se involucra la presencia de tres figuras patronales en la relación laboral. El caso de Patricia, una de las entrevistadas, ${ }^{8}$ ilustra este nuevo fenómeno: comenta que ella firmó su contrato con la empresa Servicios Integrales de RL de CV, pero labora en un call center, el cual a su vez le asignó a la campaña contratada por un banco, cuyos directivos en ocasiones se presentan a las oficinas del centro de atención para supervisar y hablar con quienes también consideran sus empleados. Así, la informante señala que deben atender a las recomendaciones de tres patrones a la vez (entrevista personal, 2015b). ${ }^{9}$ No obstante, otros de los entrevistados no tienen muy clara su relación laboral y tuvieron dificultad para indicar cuál de las tres empresas consideran que es su patrón.

En este sentido, el concepto de precariedad no se limita sólo a la inestabilidad formal (contractual), sino a aquellos empleos que pueden desaparecer, aun siendo estables en lo contractual. "La inestabilidad no se refiere a la rotación del empleo en sí misma, sino a que la desvinculación la imponga de manera unilateral el empleador" (Fernández, 2014, p. 237). Diversos testimonios coincidieron en señalar que al

8. Algunos de los entrevistados optaron por no dar su nombre y otros solicitaron no se mencionara, por lo que algunas de las entrevistas aparecen anónimas o se cambiaron los nombres. Los teleoperadores en diversos momentos de la entrevista manifestaron que firman un documento que les obliga a guardar confidencialidad. 9. La información completa de las entrevistas realizadas se presenta al inicio del apartado de bibliografía del presente artículo. Para facilidad del lector interesado, se han organizado por año y letra diferenciadora. 
momento de firmar cada contrato también se les solicita a estos trabajadores la firma de su renuncia anticipada. En el caso de Fátima, ella firmó su renuncia al momento de firmar su contrato, esto en los dos call centers en que ha laborado (entrevista personal, 2016c).

\section{Un ingreso fácil al mercado de trabajo}

Ingresar a laborar en un call center de Guadalajara es relativamente sencillo: la publicidad al respecto se encuentra en las calles, en la prensa, en Internet. Otra forma para incorporar a nuevos trabajadores a los call centers es mediante redes familiares y de amistad, que se han convertido en mecanismos informales de promoción del empleo. Además, en varios call centers existen programas que promueven e incentivan entre los trabajadores ya adscritos a la empresa el atraer a otros empleados a la corporación. En algunos call centers existe, por ejemplo, el "programa de referidos", bajo el cual si un trabajador recomienda a una persona para que se integre a la empresa, este recibe un bono de MXN 500.00 a MXN 1 000.00; incluso se permite incorporar a varias personas a la vez.

Los requisitos para el ingreso son muy similares en diversos call centers. En lo que refiere a los teleoperadores, se les solicitan conocimientos del manejo de computadora, conocimiento básicos del programa Office y Outlook, preparatoria concluida o trunca, aprobar exámenes (psicológico, de script ${ }^{10}$ de ortografía, antidoping) y en algunos casos se les requiere saber de ventas. La diferencia en los requisitos solicitados radica en el dominio del inglés: existen call centers donde este requisito es indispensable, pero no en todas las empresas se exige.

10. Script refiere a que el trabajador debe aprender el guion que la empresa utiliza para guiar lo que debe decir a los clientes. 
Cabe señalar que en estas empresas no se solicita tener experiencia previa en otro empleo entre los requisitos de ingreso, lo que hace atractivo incorporarse a este tipo de trabajo. Varios de los trabajadores afirmaron que se trataba de su primer empleo:

[...] entré a trabajar en un call center porque era mi primer empleo, y muchas empresas te piden antecedentes de dónde has trabajado y todas esas cosas, y ahí no eran necesarias. Fue el empleo que más rápido he conseguido, de que entré, [tuve] una semana de capacitación, y luego a los tres días ya estaba trabajando (entrevista personal, 20l4b).

Así, estos nuevos espacios laborales se vuelven de fácil acceso para los jóvenes o personas sin trayectoria laboral previa.

\section{Una fuerza de trabajo vulnerable}

Los directivos de estas novedosas empresas tienen clara preferencia por una mano de obra joven y soltera, pero también laboran ahí personas casadas y viudas, preferentemente mujeres. En los call centers donde se atiende a clientes extranjeros existe una franca inclinación por contratar estudiantes con dominio del idioma inglés.

En estos centros de trabajo, los empleados oscilan en edad entre los 17 y los 45 años. El porcentaje de estudiantes varía entre el 30 y el $80 \% .{ }^{11}$ Se pudo constatar la presencia de estudiantes de licenciaturas en Antropología, Sociología y Relaciones Internacionales de la Universidad de Guadalajara. Hay también un importante número de mujeres y en fechas recientes se incrementó la contratación de migrantes que retornan de los Estados Unidos, los cuales cuentan con un dominio del idioma inglés.

II. Los porcentajes de contratación de estudiantes fueron muy variables de un centro de atención a otro, sin embargo, se constata un predominio de estudiantes en la mayoría de los establecimientos estudiados. 
En los centros de atención donde no se exige el manejo de otro idioma, la fuerza de trabajo es más joven: oscila entre los 17 y 30 años de edad en un $80 \%,{ }^{12}$ y se trata mayoritariamente de estudiantes solteros, de nivel educativo medio superior. A estos trabajadores se les solicitan menos requisitos para su ingreso: tecleo rápido en computadora, excelente ortografía y buena memoria; no se exige conocimiento del inglés, pero, como se analizará a continuación, sus salarios son inferiores.

El que la fuerza de trabajo se concentre mayoritariamente en personas jóvenes, mujeres y migrantes no representa una casualidad, sino que responde a políticas no escritas, pero aplicadas en varias empresas, de seleccionar empleados que por su inexperiencia laboral o por sus trayectorias previas de trabajo en el sector informal ven en estos empleos grandes oportunidades de trabajo: "para un estudiante es buen trabajo porque la paga es buena y el horario flexible" (entrevista personal, 2015a).

Se trata de una mano de obra vulnerable, que en ocasiones no encuentra posibilidades de ocupación en otros espacios del mercado de trabajo y que está dispuesta a aceptar condiciones laborales desfavorables (Alonso Ramos, 2010). Varios de los entrevistados afirmaron haber accedido a este empleo como algo temporal (mientras concluyen sus estudios o mientras sus hijos crecen); no obstante, se detectó que algunos de ellos han permanecido en esos trabajos por varios años:

[...] estoy en un call center que no hace llamadas, [sólo] recibe. Opté por trabajar en él porque veo posibilidades de crecimiento, y pues se me abrió esta puerta. Yo iba por sólo un tiempo, unos tres meses, y ya llevo tres años [...] (entrevista personal, 2014c). 


\section{La formalidad precaria: salarios, jornadas y prestaciones}

La remuneración salarial en estas empresas es considerada por muchos de sus trabajadores como una de sus ventajas. Aun cuando algunos de los empleados entrevistados -como son los casos de Pedro y de Patricia- tienen claridad de que en otros países los salarios que pagan estas mismas compañías son más altos, esos trabajadores consideran que tomando como referencia los sueldos de otras industrias de la entidad el trabajo en los call centers no está necesariamente mal remunerado.

En los call centers en donde se exige el manejo del inglés se pagan alrededor de MXN 43.00 la hora, y el salario base mensual para un teleoperador puede llegar a los MXN 8000.00 . Existen también bonos por productividad, que van de MXN 1000.00 a MXN 2000.00 mensuales si se cumple con la meta de realizar 1000 llamadas al mes. Aunque, en opinión de Pedro, "estos bonos sólo son alcanzados por aproximadamente el 5\% del personal" (entrevista personal, 2014d). Otros testimonios coinciden en que no es fácil obtener estos sobresueldos.

Los trabajadores gozan de otras prestaciones como prima vacacional, IMSS, o INFONAVIT, y quince días de aguinaldo. En un contexto donde, como se señaló, el 45\% de los jaliscienses no tienen contratos escritos, y por ende no reciben prestaciones, estas pueden convertirse en gran atractivo para quienes solicitan un trabajo. Entre los entrevistados, los que ya tienen una trayectoria laboral y las mujeres, sobre todo aquellas que tienen hijos, son los que más valoran estos beneficios. Además, en el caso de los estudiantes de escuelas privadas, se detectó que algunos de ellos reciben becas para realizar estudios universitarios. Algunos de los call centers más grandes de la entidad realizan convenios con universidades para que sus trabajadores puedan estudiar.

A diferencia de los call centers donde se prestan servicios en otro idioma, en las empresas donde el servicio es en espa- 
ñol la remuneración salarial es menor: los salarios base para los operadores son de alrededor de MXN 3200.00 mensuales, que se pueden incrementar de acuerdo a las ventas, pero las comisiones por ellas son muy bajas. En uno de los call centers estudiados se encontró que se otorgan MXN .50 por venta, de tres a diez ventas en un día se pagan en MXN 1.00, y arriba de diez ventas se pagan en MXN 1.50.

Respecto a otras prestaciones, en los call centers en otro idioma los trabajadores cuentan con seguro social, algunos con seguro de gastos médicos mayores, bono de despensa y servicios de transporte, y horarios flexibles. En otros casos se manejan bonos por el total de ventas por día, pero no todos logran esa meta, por lo cual cada día se parte de cero y, además, los trabajadores deben estar al tanto de la duración de sus llamadas para estar dentro de sus métricas internas, eso sin contar que en ocasiones sus ventas son canceladas y sus bonos disminuyen al final, caso en el que los trabajadores no tienen ninguna información al respecto y en ocasiones esto hace que se sientan engañados por la empresa.

Las jornadas laborales en los call centers van de seis a ocho horas diarias, y también se puede trabajar tiempo extra. En opinión de los trabajadores, las jornadas son una parte atractiva del empleo: los estudiantes que trabajan presentan ante los supervisores su horario de la escuela y la empresa les establece una jornada que respeta los compromisos escolares y que además puede ser modificada de forma flexible si se requiere. No obstante, alguno de los entrevistados señaló que "sólo se respeta el horario de estudiantes de universidades como la U. de G. o el ITESO, pero no de quienes acuden a escuelas incorporadas o poco conocidas en la localidad" (entrevista personal, 2016b). Algunas empresas cuentan con servicio de transporte, tanto para la llegada como la salida del trabajo, con énfasis en el transporte para quienes salen tarde. 
También hay flexibilidad de horario para las mujeres que tienen hijos. Esthela reportó que sólo trabaja cuatro días de la semana y su horario es de medio tiempo, que el call center se encuentra en las inmediaciones de su casa y que a la salida del trabajo recoge a su niño de la guardería (entrevista personal, 2015b).

No obstante la flexibilidad que se tiene al establecer el horario, una vez que este se acuerda existe muy poca tolerancia para la llegada tarde al trabajo. Cuando un empleado se presenta después de diez minutos de su horario de entrada, se le descuenta el día; en caso de que los empleados aun así se quieran quedar a laborar, sólo reciben en pago las comisiones por lo que venden. Se detectó el caso de una empresa donde al momento de firmar el contrato se le solicita al trabajador sincronizar su reloj con el de la compañía para evitar malos entendidos respecto a los horarios. Únicamente los trabajadores que se transportan en el camión de la empresa tienen dispensa si hay retraso en la llegada, lo que habla de un estricto control sobre la fuerza de trabajo.

\section{Control sobre el trabajo}

El control sobre la fuerza laboral y los servicios que se prestan en los call centers se ejerce a través de un equipo de supervisores, quienes vigilan directamente a los empleados, pero el mecanismo más sofisticado y efectivo se presenta por la vía de las herramientas de trabajo: las computadoras. El control es tan rígido que algunos autores han llegado a sugerir que se trata de un nuevo control de tipo taylorista (Micheli Thirión, 2007).

Las computadoras programadas con las bases de datos de los clientes son las que controlan las llamadas de entrada y salida y los tiempos de duración de cada una de ellas. En la pantalla generalmente aparece el script y el tiempo de duración de la llamada. En la mayoría de los call centers se 
les da a los empleados un máximo de cinco minutos como tiempo para lograr vender o colocar una promoción, y en caso de no hacerlo en ese tiempo, una vez que se ha insistido de distintas maneras, el operador debe buscar el momento de terminar la conversación o en su defecto la máquina lo desconecta automáticamente. Aunque el número de llamadas puede variar de un centro a otro, se encontró que por lo regular se solicita que un trabajador realice no menos de cien por día, aunque se registraron casos que rebasan significativamente dicha cantidad.

Todas las llamadas son grabadas para que los supervisores puedan revisarlas y realizar una evaluación de las mismas, también para buscar fórmulas exitosas de colocación de productos, revisar errores en la promoción o incluso buscar si se compartió información confidencial que el cliente no debería conocer. ${ }^{13}$ Así es como se realiza un segundo control sobre la acción de los empleados.

Los supervisores pueden también establecer una conexión en directo con las llamadas para evaluar in situ el trabajo del operador. En algunas empresas, además de los supervisores, están los líderes de proyecto o campaña, quienes vigilan constantemente el trabajo de los empleados, pero en especial están al tanto del cumplimiento de las metas programadas, para lo cual pueden exigir cambios en los ritmos o formas de trabajo. A pesar de que son varias jerarquías que pueden adoptar diferentes nombres, el supervisor fue la figura más reconocida por los operadores.

Otra de las formas en que se manifiesta el control sobre los empleados en algunos de los call centers tiene que ver con la estandarización de las formas de vestir (formal, no mezclilla, no tenis, no minifalda). Los piercings y los tatuajes son motivo de despido en algunas de estas empre-

13. Para evitar estas situaciones, las empresas hacen que los trabajadores firmen una cláusula de manejo confidencial de los datos que utilizan, y no respetar dicha cláusula es considerado una causal de despido. 
sas, aunque vale la pena aclarar que estas políticas no se comparten en todos los centros de atención: se detectaron algunos que permiten que sus empleados vistan con comodidad y porten tatuajes, especialmente donde se emplea a migrantes retornados. ${ }^{14}$ Este hecho vuelve el trabajo más atractivo para los jóvenes.

También se hace visible el control a través de otras medidas, como no permitir dentro de las áreas de trabajo celulares, libros, cuadernos, alimentos, etc., ya que sólo se puede tener una botella de agua, que debe ser transparente para que se pueda verificar qué tipo de líquido se está ingiriendo.

En algunos call centers se prohíben las relaciones afectivas entre los empleados. Los trabajadores perciben el clima laboral como tenso, frío, hostil y distante, descripción que corresponde con las primeras imágenes de quienes investigaron el trabajo en los call center, en las primeras etapas de su aparición. No obstante, conviene señalar que también se encontraron centros de atención donde se propicia un mejor ambiente laboral, y donde según versión de algunos trabajadores jóvenes el ambiente es "hasta divertido" (entrevista personal, 2016a). ${ }^{15}$ Estas imágenes se asemejan a las condiciones de trabajo descritas por Meoño Artigas (2011) en los call centers de Guatemala, donde las empresas promueven fiestas y diversas celebraciones a las que los jóvenes acuden, muchas de las veces disfrazados. De ahí la necesidad de avanzar a estudios de mayor profundidad

14. En uno de los centros de atención, uno de los más grandes que se ha instalado en la ZMG, los trabajadores se organizaron y firmaron una petición a la empresa para poder vestir ropa de mezclilla y calzar tenis. "La empresa aceptó la petición, pero prohibió el uso de faldas cortas a fin de que las empleadas no distrajéramos a los supervisores" (entrevista personal, 2015d).

15. El propiciar por parte de las gerencias de los call centers un ambiente de trabajo juvenil y divertido fue documentado como parte de una estrategia del "dispositivo de control" hacia los trabajadores en una investigación realizada por Abal Medina sobre las empresas instaladas en Argentina. Este argumento se trata a profundidad en otro trabajo que aún se encuentra en proceso de revisión. 
que muestren la complejidad y la heterogeneidad de cómo se vive el trabajo en estos nuevos empleos.

Sin embargo, algo que sí se manifestó como una constante son los fuertes controles para evitar las posibles uniones de los trabajadores con miras a la formación de sindicatos o asociaciones de defensa de sus derechos.

\section{Relaciones laborales}

En lo general, los trabajadores de los centros de atención de la ZMG no están afiliados a sindicatos. Todos los teleoperadores entrevistados comentaron no tener conocimiento de contar con algún tipo de organización gremial para la defensa de sus derechos laborales; también afirmaron que no reciben descuentos por este concepto en su nómina de pago y que sus contratos de trabajo son individuales y en su mayoría por tiempo u obra determinada.

La relación con los directivos de la empresa se da a través de los supervisores. La mayor parte de los entrevistados señalaron que la relación con ellos es buena. Los supervisores o coachs dependen en cierta medida del buen desempeño de los teleoperadores para conservar su empleo. En general, se señala que las políticas de las empresas buscan motivar a los equipos para conseguir las metas de las campañas, más que sólo dar simples regaños. Otros trabajadores afirman que la motivación que reciben de los supervisores para realizar su trabajo puede volverse incomoda, principalmente en momentos cuando las campañas no dan el resultado deseado, que también se padece de cierta discrecionalidad de su parte y que en ocasiones sí se llegan a presentar actitudes de hostigamiento hacia algunos empleados o la insinuación de tipo sentimental hacia las mujeres. Finalmente, hay quienes se quejan de un ambiente de trabajo poco franco:

Yo noto un ambiente muy hipócrita con todos, es muy hipócrita ahí, porque la empresa te dice: "nosotros tenemos valores de respeto, de 
responsabilidad", $y$ te tratan de infundir dichos valores, pero precisamente es en las relaciones con los supervisores que te das cuenta que, pues, no es así. lgual a [sic] tu supervisor le caes "gordo", pero como nomás tiene la obligación de tratarte bien, pues lo hace. No es una relación pura y sana, yo diría [que] no es buena la relación (entrevista personal, 20I4e).

Por otra parte, la temporalidad tanto de los contratos de trabajo como de las campañas contratadas por los clientes hace que muchos despidos no tengan una mayor consecuencia que la liquidación por el término de la relación laboral o la materia de trabajo. En otros casos, se busca la forma de presionar a la firma de la renuncia para evitar demandas.

De acuerdo a los entrevistados, los malos tratos hacia ellos procedían de forma más regular de parte de los clientes que de los supervisores. Los clientes que no desean comprar los servicios que vende la empresa no sólo se niegan a entablar un diálogo con el vendedor, sino que en muchas ocasiones los insultan o les cuelgan el teléfono: "La relación con los clientes era agresiva" (entrevista personal, 2014a). Entre otras cosas, varios de los entrevistados mencionaron el uso de palabras altisonantes en su contra, el racismo hacia los mexicanos por clientes extranjeros y la descalificación de sus saberes, eso sin dejar de mencionar que la empresa les señala que el cliente siempre tiene la razón.

La intensidad del trabajo en constante supervisión y los continuos malos tratos de los clientes hacen que muchos trabajadores declaren tener un alto nivel de estrés laboral: “creo que es de los empleos más estresantes que hay, sí, sí es estresante. Hay que aprender a lidiarlo y mantener una concentración y un estado estable" (entrevista personal, 2014c). 


\section{Rotación y ascensos de la fuerza de trabajo}

La alta rotación de los trabajadores es una de las características principales de estos empleos. La rotación de personal oscila entre 50 y $60 \%$ anual en la India, y en algunos call centers de Argentina alcanza 100\% (Hualde, Tolentino y Jurado, 2014, p. 221). En Jalisco, la rotación de los teleoperadores es de alrededor de 35\% (entrevista personal, 2015e), que aunque es más baja que en los países mencionados, se considera alta en el contexto local.

No obstante, entre los entrevistados a pesar de la alta rotación se presentaron varios casos de trabajadores que se han adaptado a las condiciones laborales existentes en estas empresas y han continuado trabajando en ellas por varios años, contando con una antigüedad de alrededor de cinco años. Cabe señalar que no se encontró ningún trabajador con antigüedad superior a seis años.

Algunos de los beneficios que se derivan de la alta rotación de personal es que los trabajadores reciben un bono de MXN $6500.00^{16}$ por permanecer en la empresa después de seis meses de haber ingresado. Además, los trabajadores que superan este lapso tienen la posibilidad de reintegrarse aun después de haberse retirado por alguna causa de tipo personal.

En cuanto a las posibilidades de ascenso, estas van unidas a la antigüedad y al buen desempeño laboral, evaluado principalmente con la obtención de altas calificaciones en sus llamadas. En algunas empresas las oportunidades de ascenso se presentan anualmente, y al estar relacionadas con la calidad del trabajo sirven como un aliciente para la continuidad laboral y para mejorar el rendimiento de los trabajadores. No obstante, varios de los entrevistados afirman que los ascensos sólo se contemplan para los teleoperadores que trabajan la jornada completa, y no de medio tiempo.

16. Este bono se otorga en una de las empresas transnacionales más importantes que se ha instalado en la localidad. 
En otros centros las promociones no están formalizadas y dependen mucho de la discrecionalidad de los supervisores.

\section{Condiciones de las áreas de trabajo}

En general, las instalaciones de las empresas más grandes se encuentran en amplias bodegas o galerías, las cuales pueden ser adaptaciones o construidas ex profeso para los call centers. Algunos de los entrevistados comentaron sobre un centro de atención de la ZMG que tiene café y salas de juego y de relajación disponibles para los empleados, pero no es lo común. Existen otras empresas pequeñas que están instaladas en espacios reducidos, como casas u oficinas adaptadas para ello, y que los trabajadores describieron como espacios "lúgubres y fríos" (entrevista personal, 2015c). Para su operación, estas empresas necesitan de una constante fuente de energía eléctrica, acceso a líneas telefónicas y al Internet, ello debido a que los equipos de trabajo, generalmente servidores de Internet, computadoras y diademas telefónicas, requieren de ello. En los grandes call centers estos equipos son modernos, están en buenas condiciones y son suficientes para todos los trabajadores, pero en otros, por lo regular los pequeños, el equipo se encuentra en mal estado y los trabajadores deben disputar por conseguir un equipo dónde laborar.

La necesidad de mantener en estado óptimo estas herramientas de trabajo hace que se privilegie la máquina por encima del hombre, por lo cual la luz y el clima deben ser artificiales ante la necesidad de prevenir el desgate del equipo y mantener una temperatura adecuada para las computadoras y servidores. La luz artificial permite que los equipos no se desgasten por la radiación solar, pero también mantiene atento al operador, mientras que el clima frío, además de favorecer al no sobrecalentamiento de los equipos informáticos, permite que el operador este en una constante alerta en el horario de trabajo. 
Por su parte, el mobiliario tiende a ser poco cómodo, pues se trata de simples estaciones de trabajo compuestas por cubículos pequeños con una mesa, una silla, una computadora y diadema telefónica, sin espacios para descansar la vista, y donde las posibilidades de interacción con otros trabajadores son mínimas. Ante la incomodidad, los trabajadores han manifestado que se estiran mientras están sentados o se ponen de pie, pero en ambas situaciones continúan atendiendo llamadas. Cuando acuden al baño, les cuentan el tiempo: la duración permitida de estos recesos va de los dos a los diez minutos, según la empresa de que se trate.

Algunos call centers tienen comedor para los trabajadores que laboran jornadas más largas, pero se impide que todos vayan al mismo tiempo. En esos comedores se tienen máquinas expendedoras de bebidas o golosinas, o pequeños locales de venta de café y alimentos. Las máquinas y los expendios pueden ser propiedad de los call centers o concesiones a terceros. Algunas trabajadoras se quejan del escaso tiempo que se otorga para ingerir sus alimentos:

[...] sólo nos permitían ir al baño una vez, y nos dan de cinco a veinte minutos para comer, dependiendo de las personas que asistan. Una vez nos tocaron cinco minutos para el baño y cinco para comer. El comedor estaba cuatro pisos abajo por la escalera y no podíamos usar el elevador. Este sólo se permite para subir [...], ise imagina? (entrevista personal, 2016a).

Sólo los call centers más modernos cuentan con las instalaciones apropiadas para su desarrollo, e incluso tienen beneficios extras como salas de descanso para sus empleados. Sin embargo, los trabajadores siguen manifestando que el frío y los ambientes artificiales, además del manejo de los equipos, les han generado enfermedades laborales como dolores musculares en las extremidades superiores, especialmente en las manos, así como alergias, además de 
daños relacionados con la postura del cuerpo porque no cuentan con un mobiliario ergonómico.

\section{Conclusiones}

El avance de investigación realizado hasta este momento permite pensar que en los empleos actuales, en especial los ofrecidos por los call centers, se presencian nuevas formas de precarización, con la especificidad de que este proceso, a diferencia del que se presenció en décadas pasadas, se vive en el sector formal de la economía.

Las características que asume esta flexi-precariedad son, entre otras:

- Que en la mayor parte de estos empleos los trabajadores no pertenecen a un sindicato o afirman no estar enterados de su existencia; no hay un ejercicio de este derecho laboral.

- Que la temporalidad del empleo se ha relativizado con la existencia de varios contratos temporales de trabajo que alargan la obtención de la definitividad del mismo y que facilitan el despido por parte de los patrones, generalmente argumentando la falta de materia de trabajo, la conclusión de las campañas o el incumplimiento de metas. Esto genera inestabilidad e incertidumbre laboral.

- Que el trabajo por cumplimiento de metas en donde las buenas remuneraciones sólo se alcanzan con base en el rendimiento y la buena evaluación de los servicios prestados parece rememorar al trabajo a destajo, con la diferencia de que en los servicios de atención al cliente las ventas no se remuneran si no se alcanza el tope establecido por la empresa. Es decir, el salario también se ha visto afectado por las condiciones de los contratos de trabajo y las políticas de los call centers. $\mathrm{Al}$ respecto, es común que se pague un salario base y que los bonos 
de productividad se repartan de forma poco clara, de manera que si se cumplen las metas se tiene derecho al bono, y de que cuando no se cumplen, además de no recibir el bono extra, los trabajadores comienzan las nuevas jornadas desde cero, sin respetar el trabajo ya acumulado previamente.

- Sumado a lo anterior, hay que señalar que en estas empresas el control del trabajo se ha vuelto más focalizado y estricto: se realiza sobre cada uno de los trabajadores a través del monitoreo de una computadora, la cual después de cierto tiempo desconecta de forma automática el servicio que ofrece el trabajador y le da una nueva orden de trabajo, al tiempo que almacena las conversaciones para efecto de futuras revisiones por parte de los supervisores.

Asimismo, las condiciones para la realización del trabajo no son las más adecuadas, puesto que debido a las necesidades de la máquinas y tecnologías utilizadas, generalmente computadoras, servidores de Internet y líneas telefónicas, se privilegian las condiciones de temperatura y acomodo de las máquinas por encima de las necesidades de los individuos, esto en materia de clima, ergonomía y necesidades de espacio y movilidad.

Finalmente, se puede señalar que las situaciones anteriormente enumeradas dan cuenta de una precarización de las condiciones laborales de los trabajadores de los call centers y de una alta vulnerabilidad en el respeto de sus derechos laborales, lo cual permite afirmar lo ya señalado: este tipo de empleos genera una nueva clase de precariedad laboral, ahora ya legislada por el Estado en la nueva Ley Federal del Trabajo. 
Bibliografía

(20I4a). Entrevista personal a Anónima, teleoperadora, realizada por J. D. Zazueta en el marco del proyecto "Nuevas formas de precarización laboral en el sector de los servicios especializados. El caso de los call centers y sus trabajadores en la ZMG”. Universidad de Guadalajara, Guadalajara, México.

(20I4b). Entrevista personal a Carlos, teleoperador, realizada por J. D. Zazueta en el marco del proyecto "Nuevas formas de precarización laboral en el sector de los servicios especializados. El caso de los call centers y sus trabajadores en la ZMG". Universidad de Guadalajara, Guadalajara, México.

(20I4c). Entrevista personal a Juan, teleoperador, realizada por J. D. Zazueta en el marco del proyecto "Nuevas formas de precarización laboral en el sector de los servicios especializados. El caso de los call centers y sus trabajadores en la ZMG". Universidad de Guadalajara, Guadalajara, México.

(20I4d). Entrevista personal a Pedro, realizada por E. de la Paz Hernández en el marco del proyecto "Nuevas formas de precarización laboral en el sector de los servicios especializados. El caso de los call centers y sus trabajadores en la ZMG". Universidad de Guadalajara, Guadalajara, México.

(20I4e). Entrevista personal a Sandra, teleoperadora, realizada por J.D.Zazueta en el marco del proyecto "Nuevas formas de precarización laboral en el sector de los servicios especializados. El caso de los call centers y sus trabajadores en la ZMG". Universidad de Guadalajara, Guadalajara, México.

(20I5a). Entrevista personal a Gabriela, teleoperadora, realizada por E. de la Paz Hernández en el marco del proyecto "Nuevas formas de precarización laboral en el sector de los servicios especializados. El caso de los 
call centers y sus trabajadores en la ZMG”. Universidad Bibliografía de Guadalajara, Guadalajara, México.

(20I5b). Entrevista personal a Esthela, teleoperadora, realizada por E. de la Paz Hernández en el marco del proyecto "Nuevas formas de precarización laboral en el sector de los servicios especializados. El caso de los call centers y sus trabajadores en la ZMG". Universidad de Guadalajara, Guadalajara, México.

(20I5c). Entrevista personal a Maricela, teleoperadora, realizada por E. de la Paz Hernández en el marco del proyecto "Nuevas formas de precarización laboral en el sector de los servicios especializados. El caso de los call centers y sus trabajadores en la ZMG". Universidad de Guadalajara, Guadalajara, México.

(20I5d). Entrevista personal a Patricia, teleoperadora, realizada por E. de la Paz Hernández en el marco del proyecto "Nuevas formas de precarización laboral en el sector de los servicios especializados. El caso de los call centers y sus trabajadores en la ZMG". Universidad de Guadalajara, Guadalajara, México.

(20I5e). Entrevista personal a Lic. Joel, coach, realizada por J. J. Morales en el marco del proyecto "Nuevas formas de precarización laboral en el sector de los servicios especializados. El caso de los call centers y sus trabajadores en la ZMG". Universidad de Guadalajara, Guadalajara, México.

(20I6a). Entrevista personal a Camarena, teleoperadora, realizada por E. de la Paz Hernández en el marco del proyecto "Nuevas formas de precarización laboral en el sector de los servicios especializados. El caso de los call centers y sus trabajadores en la ZMG". Universidad de Guadalajara, Guadalajara, México.

(2016b). Entrevista personal a Edgar, teleoperador, realizada por E. de la Paz Hernández en el marco del proyecto "Nuevas formas de precarización laboral en el sector de 
Bibliografía los servicios especializados. El caso de los call centers y sus trabajadores en la ZMG”. Universidad de Guadalajara, Guadalajara, México.

(20I6c). Entrevista personal a Fátima, teleoperadora, realizada por E. de la Paz Hernández en el marco del proyecto "Nuevas formas de precarización laboral en el sector de los servicios especializados. El caso de los call centers y sus trabajadores en la ZMG". Universidad de Guadalajara, Guadalajara, México.

Alonso Ramos, J.A. (20I0). ¿Tayloristas eminentes? los trabajadores de los call centers en México (tesis de maestría inédita). El Colegio de la Frontera Norte: Tijuana.

Arteaga García, A.,y Micheli Thirión,J.(2010).“Trabajador@s en call centers: ¿flexibilidad vs. ciudadanía”, en A. Arteaga García (coord.), Trabajo y ciudadanía, una reflexión necesaria para la sociedad del siglo XXI (Pp. I77-2 I5). México: Miguel Ángel Porrúa, UAM.

Da Cruz, M., y Fouquet, A. (2010). "La figura del operador mundializado: jóvenes trabajadores en los call centers de Monterrey", en C. Contreras, V. Zúñiga, T. Bloss, et al. (coords.), La globalización permanencia y cambio en el área Metropolitana de Monterrey UANL (pp. 433-456). México: Colegio de la Frontera Norte.

De la Garza, E. (20II). “Introducción. Construcción de la identidad y acción colectiva entre trabajadores no clásicos como problema", en E. de la Garza Toledo (coord.), Trabajo no clásico, organización y acción colectiva. Tomo ॥ (pp. I I-22). México: Plaza y Váldez, UAM.

De Oliveira, O. (2006). “Jóvenes y precariedad laboral en México”. Papeles de Población, I2(49), 37-73.

Del Bono, A., y Bulloni, N. (2008). “Experiencias laborales juveniles. Los agentes telefónicos de call centers offshore en Argentina". Trabajo y Sociedad, IX (I0). Recuperado de: http://www.unse.edu.ar/trabajoysociedad/ DEL_BONO. pdf

\section{8}


Del Toro, I. (2016). "Precio de la canasta básica incrementó 4.8\% al cerrar junio en ZMG”. Recuperado de: http:// www.udg.mx/es/noticia/precio-de-la-canasta-basicaincremento-48-al-cerrar-junio-en-zmg

El Informador (20I4). "El outsourcing dentro de la nueva Ley Federal del Trabajo. Comentada y concordada”. Recuperado de: http://www.noticierofiscal.com/index.php/ categorias/outsourcing//6-especiales/66I-el-outsurcingdentro-de-la-nueva-ley-federal-del-trabajo.html

ENOE (20 I3). Resultados de la Encuesta Nacional de Ocupación y Empleo en el primer trimestre de 2012 para el Estado de Jalisco. INEGI. Boletín de Prensa I6///2. Recuperado de: www.inegi.org.mx

Escobar Salazar, A. P. (20I2). Las fábricas de la charla en Santiago de Chile: materialidad y subjetividad del trabajo en los call centers (tesis doctoral inédita). Universidad Autónoma de Barcelona: Barcelona.

Fernández, M. (20I4).“'Dimensiones de la precariedad laboral: un mapa de las características del empleo sectorial en Argentina”. Cuadernos de Economía, XXXIII(62), 23I-258.

Garza, G. (20I I). Visión comprensiva de la distribución territorial del sector servicios en México. México: El Colegio de México.

Guadarrama, R., Hualde, A., y López, S. (coords.) (20I4). La precariedad laboral en México. Dimensiones, dinámicas y significados. México: El Colegio de la Frontera Norte, Universidad Autónoma Metropolitana.

Hualde, A., y Serrano, A. (2005). "La calidad del empleo de asalariados con educación superior en Tijuana y Monterrey. Un análisis cuantitativo". Revista Mexicana de Investigación Educativa (RMIE), I0(25), 345-374.

Gomis, R., Jurado, M., et al. (20I I). "Contratos y salarios en los operadores de call centers. Análisis de la precariedad y heterogeneidad en tres ciudades mexicanas" (ponencia preparada para el Congreso de la AMET 
Bibliografía

20 l I). Mérida, Yucatán. Recuperado de: http://www. izt.uam.mx/sotraem/Documentos/AMET20II/REC/ TEXTO/0I/0I_I2.pdf

Tolentino, H., y Jurado, M. A. (20I4). "Trayectorias laborales en los call centers: ¿empleos sin futuro?” en R. Guadarrama, A. Hualde y S. López (coords.), La precariedad laboral en México. Dimensiones, dinámicas y significados (pp. 22 I-256). México: El Colegio de la Frontera Norte, Universidad Autónoma Metropolitana.

IMT (20 I3). Censo Nacional de Agencias 20 I 3. Recuperado de: http:// www.imt.com.mx/Estudios-Realizados

Lindón, A. (2003).“'La precariedad laboral como experiencia a través de la narrativa de vida". Gaceta Laboral, 9(3), 333-352.

Lisdero, P. (2009). “Visibilidad y conflicto. Algunas imágenes de las organizaciones de trabajadores de call centers en Córdoba”. Boletín Onteaiken, (7), 59-72.

López Pedroza, M. G. (2016). "Veinte años de la flexibilidad laboral y el empleo precario en Jalisco" (ponencia presentada en el 4to. Congreso Latino Americano de Ciencias Sociales). Zacatecas, México.

Meoño Artigas, L. P. J. (20I I). Los trabajadores de la industria de call centers de la ciudad de Guatemala:diferenciación social y representaciones sobre el trabajo (tesina de maestría inédita). Universidad Autónoma Metropolitana: México. Micheli Thirión, J. (2007). "Los call centers y los nuevos trabajos del siglo XXI”. Revista CONfines, (3/5), 49-58.

(20I2)."El sector de call centers : estructura y tendencias.Apuntes sobre la situación de México”. Frontera Norte, 24(47), I45-169.

Montarcé, I. (20I I)."Del otro lado del teléfono: identidad y acción colectiva en call centers de la Ciudad de México", en E. de la Garza Toledo (coord.), Trabajo no clásico, organización y acción colectiva. Tomo II (Pp. 69-122). México: Plaza y Váldez, UAM. 
Hernández, J., Garabito, G., et al. (20I2).“CondicioBibliografía nes de trabajo y organización en algunas ocupaciones 'atípicas': call centers, Walmart, Macdonald's, extras de televisión, ambulantes del centro histórico, bagoneros, tianguistas, microbuseros, taxistas, metrobuseros", en E. de la Garza (coord.), La situación del trabajo en México, 20I2. El trabajo en la crisis (pp. 345-394). México: Plaza y Váldes, Universidad Autónoma Metropolitana.

Mora, M. (2005). "Ajuste y empleo. Notas sobre la precarización del empleo asalariado”. Revista Ciencias Sociales, (I08), 27-40.

Piore, M., y Sabel, C. F. (1990). La segunda ruptura industrial. España:Alianza.

Rodgers, J., y Rodgers, G. ( 1989). Precarius jobs market regulation. Suiza: International Institute for Labour Studies, Free University of Brussels.

Rosenfield, C. (2007). "Paradoxos do capitalismo e trabalho em call centers : Brasil, Portugal e CaboVerde”. Caderno $\mathrm{CRH}, 20(5 \mathrm{I}), 447-462$.

Taylor, P., y Bain, P. M. (2005). "India calling to the far away towns: the call center labour process and globalization". Work, Employment and Society, 19(2), 26I-282.

Viadana, C.A., Morales, O., Cavallo, M., et al. (20I0).“Comunicación intercultural en el ámbito laboral: los call centers bilingües en la ciudad de Rosario" (ponencia presentada en las Decimoquintas Jornadas de Investigaciones en la Facultad de Ciencias Económicas y Estadísticas). Universidad Nacional Paraíso, Panamá.

Zapata, F. (2005). Tiempos neoliberales en México. México: El Colegio de México. 